Mediterránea Ser. Biol. (1989), n. o 11. Pág. 35-46

\title{
SEGREGACIÓN Y SINCRONISMO DE LA AVIFAUNA ACUÁTICA EN LA LAGUNA DE FUENTE PIEDRA (MÁLAGA) EN EL PERÍODO REPRODUCTOR
}

\author{
por \\ J. M. SÁNCHEZ, M. BLASCO, C. DE LA CRUZ \& E. DA SILVA
}

\section{RESUMEN}

En la Laguna de Fuente Piedra (Málaga, España) se estudian las preferencias de las diferentes especies acuáticas para ubicar sus nidos, detectándose una competencia interespecífica por la ocupación del espigón central y espigones del NE, en especial referente al Flamenco común y Pagaza piconegra, que son además las especies más numerosas. Igualmente se observa una segregación de especies en función de sus preferencias por aguas salobres o no, detectándose asociaciones importantes como es la formada por Láridos y Recurviróstridos. Por su parte, el sincronismo de la fenología reproductora de las especies es pobre, no superando en ningún caso el $40 \%$.

\section{SUMMARY}

In Fuente Piedra lagoon (Málaga, Spain) we studied the favorite places the different aquatic species choose to lie their nests and we noted an interspecific competence in order to occupy the Central pint and the points in the NE. We found this competence specially remarkable in Greater Flamingo and Gull-billed tern, which are also the most numerous species. We also observed a segregation of the species regarding their preferences towards brackish or not brackish water and important associations as that between Laridae and Recurvirostridae. With regards to the sincronicity in the reproductive fenology of these species, we found it poor; it never exceeded $40 \%$.

\section{INTRODUCCIÓN}

La laguna de Fuente Piedra (Málaga), una de las zonas húmedas más relevantes de la Península Ibérica, se dío a conocer principalmente por la colonia de Flamencos (Phoenicopterus ruber, L.) que allí nidifica. Numerosas investigaciones han tenido como material de estudio esta colonia (Valverde, 1964; Studer-Thiersch, 1968; Antúnez et al, 1979; Blasco et al, 1979; Vargas et al, 1983; Sánchez et al, 1985). Por el contrario, a otras especies se les ha prestado 
poca o nula atención; así Vargas et al (1983) realizan un estudio sobre la comunidad de vertebrados que puebla la laguna, Antúnez et al (1977) sobre el régimen alimentario de la Pagaza piconegra (Gelochelidon nilotica, Gm.) y Amat (1984) la incluye dentro de un amplio estudio sobre la comunidad de acuáticas de las lagunas andaluzas. Pero sin duda, resultaba conveniente realizar un estudio sobre la avifauna que habitualmente se reproduce en su entorno y que abarcase el tipo de asociaciones que presentan, el grado de utilización de las distintas áreas de nidificación, la segregación y sincronismo que presenta las diferentes especies. Estos son los objetivos que hemos intentado alcanzar.

\section{MATERIAL Y MÉTODOS}

Previamente a la primavera se delimitaron unas áreas de similar fisionomía (Figura 1):

Área 1.-Zona del Suroeste formada por una serie de isletas, que se ponen en contacto con la orla durante las primeras fases de la primavera. Botánicamente se caracteriza por las asociaciones Frankeniataeae pulverulentae Rivas Martínez, 1975 y Arthronemetea fruticosi BrBl \& Tx, 1943.

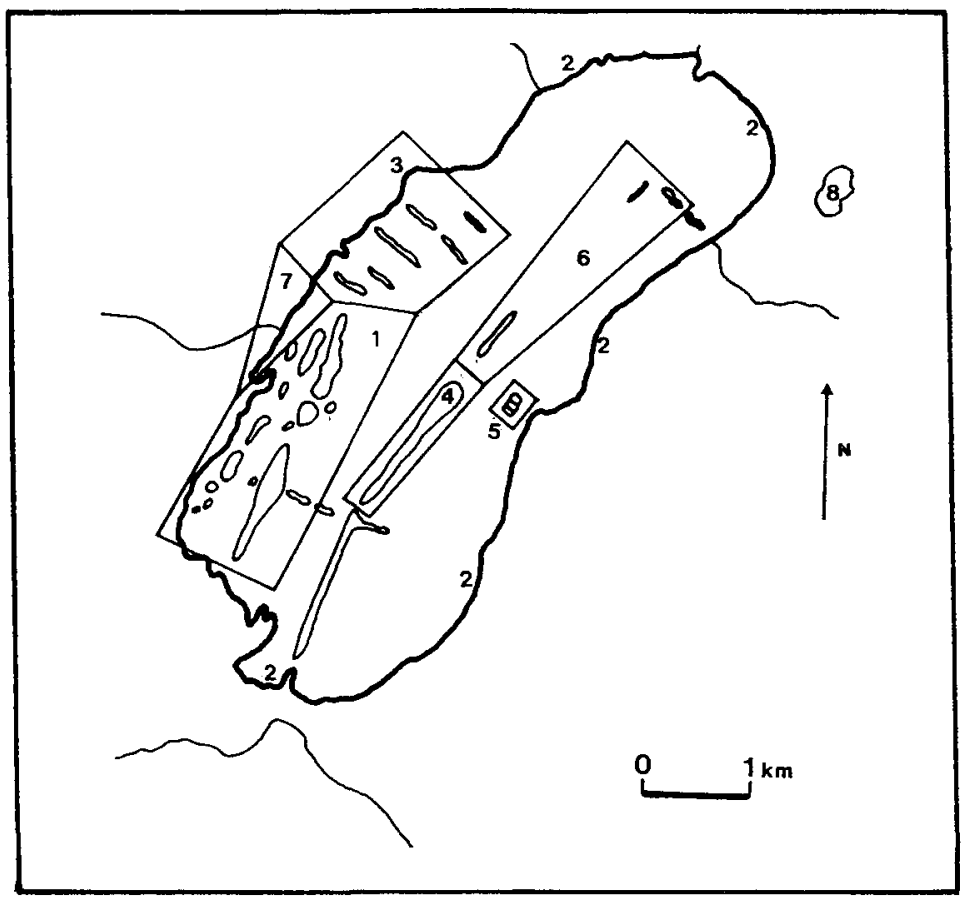

Figura 1.-Mapa de la laguna de Fuente Piedra en la que quedan delimitadas las áreas descritas en el presente estudio. 
Área 2.-Región compuesta por la orla de la laguna y que carece de vegetación.

Área 3.-Formada por los espigones del Oeste, cuya vegetación, compuesta por las asociaciones anteriormente señaladas es rala.

Área 4.--Segundo tramo del espigón central, que recorre longitudinalmente la masa de agua. Está cubierto de vegetación, siendo ésta menos abundante a medida que nos aproximamos a su región distal.

Área 5.-Constituidas por las salinas al este del Espigón Central. Se trata de una formación pedregosa sobre la que se asientan Arthronemum glaucum (Delille), Suaeda vera (Gm.) y Suaeda splendens (Pourret).

Área 6.-Formada por una serie de espigones próximos a la orilla Este, que no se ponen en contacto con ella hasta que la laguna no está prácticamente seca. La vegetación es dispersa y compuesta por las comunidades ya citadas.

Área 7.-Comprende la zona que queda encharcada en la desembocadura del arroyo de los Arenales y que da lugar a una superficie cubierta por aguas dulces. Su vegetación está formada por Phragmites communis, Juncus subulatus, Juncus bufonis y Scirpus maritimus.

Área 8.-La laguna próxima al pueblo donde son vertidas las aguas residuales. La vegetación es similar a la anterior.

Una vez que la avifauna fue ocupando sus zonas de reproducción, se llevaron a cabo visitas semanales. En éstas se realizaron observaciones sobre el comportamiento reproductor y territorialidad de las diferentes parejas y especies, lo que ayudó a la posterior búsqueda de los nidos. Estos fueron calculados por conteo directo dado las característcas del entorno y de las especies. En el caso de Phoenicopterus ruber esto no fue posible, utilizándose fotografías aéreas de las colonias.

Con los datos obtenidos se calculó el índice de amplitud (Isenmann, 1979), el de solapamiento (Cody, 1974), la diversidad (Shannon-Weaver, 1948) a cada una de las áreas y el índice de dominancia de Lebreton (en Thevenot, 1982). Para el estudio del solapamiento fenológico se utilizó el índice de Pianka (Lawlor, 1980), aplicado éste a las distintas fases de la reproducción. Dicha fenología fue tipificada atendiendo a las siguientes variables: fenología de inmigración de la especie, ocupación de la colonia, primeras puestas, primeras y últimas eclosiones, primeros abandonos de las colonias y del entorno. Igualmente se obtuvieron los períodos de prepuesta y cría.

\section{RESULTADOS}

La especie más abundante como reproductora fue Phoenicopterus ruber, con un total de 2.500 parejas (Tabla 1 ), seguida de Gelochelidon nilotica con 261 parejas, mientras que el resto presentan contingentes muy inferiores.

En cuanto a la colonización de las distintas áreas, hay que señalar que el número de especies, así como el de parejas, fue máximo en el espigón central (área 4), con 2.623 parejas pertenecientes a siete especies, siendo la más abundante Phoenicopterus ruber. 
TABLA I

\begin{tabular}{|c|c|c|c|c|c|c|c|c|c|}
\hline $\begin{array}{l}\text { Áreas } \\
\text { Espec. }\end{array}$ & 1 & 2 & 3 & 4 & 5 & 6 & 7 & 8 & \\
\hline $\mathrm{Fa}$ & & & & & & & & $\begin{array}{ll}5 & \\
& \\
& 100 \\
\end{array}$ & 5 \\
\hline $\mathrm{Gc}$ & & & & & & & $\begin{array}{l}1 \\
\quad 25 \\
\end{array}$ & 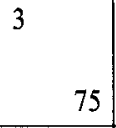 & 4 \\
\hline $\mathrm{Ap}$ & $\begin{array}{|ll|}16 & \\
& 50 \\
\end{array}$ & $\begin{array}{ll}8 & \\
& \\
& 25 \\
\end{array}$ & $\begin{array}{ll}2 & \\
& \\
& 6.2 \\
\end{array}$ & $\begin{array}{ll}2 & \\
& 6.2 \\
\end{array}$ & & & $\begin{array}{ll}3 & \\
& 9.3 \\
\end{array}$ & $\begin{array}{|ll|}1 & \\
& \\
& 3.1 \\
\end{array}$ & 32 \\
\hline $\operatorname{Pr}$ & & & & $\begin{array}{r}2.500 \\
100 \\
\end{array}$ & & & & & 2.500 \\
\hline $\mathrm{Ca}$ & & $\begin{array}{l}7 \\
\quad 70.1 \\
\end{array}$ & $\begin{array}{r}14 \\
\quad 20.9 \\
\end{array}$ & $\begin{array}{ll}1 & \\
& 1.5 \\
\end{array}$ & $\begin{array}{ll}3 & \\
& 4.6 \\
\end{array}$ & & 2 & & 67 \\
\hline $\mathrm{Hh}$ & & $\begin{array}{l}2 \\
\quad 16.6 \\
\end{array}$ & & & & $\begin{array}{ll}2 & \\
\quad 166 \\
\end{array}$ & \begin{tabular}{|r}
4 \\
\\
$\quad 33.2$ \\
\end{tabular} & $\begin{array}{|ll|}4 & \\
& \\
& 33.2 \\
\end{array}$ & 12 \\
\hline $\mathrm{Ra}$ & & $\begin{array}{r}8 \\
\quad 11.3 \\
\end{array}$ & $\begin{array}{l}9 \\
12.7 \\
\end{array}$ & $\begin{array}{r}37 \\
\quad 52.1 \\
\end{array}$ & $\begin{array}{ll}2 & \\
& 2.8 \\
\end{array}$ & $\begin{array}{l}9 \\
12.7 \\
\end{array}$ & \begin{tabular}{|ll}
$\begin{array}{l}6 \\
\end{array}$ & \\
& 8.5 \\
\end{tabular} & & 71 \\
\hline $\mathrm{Tt}$ & & & & & & & \begin{tabular}{|ll}
3 & \\
& 60 \\
\end{tabular} & $\begin{array}{|ll|}2 & \\
& \\
& 40 \\
\end{array}$ & 5 \\
\hline $\mathrm{Lr}$ & & & $\begin{array}{rr}3 & \\
& 5.1 \\
\end{array}$ & $\begin{array}{l}16 \\
\quad 27.1 \\
\end{array}$ & & $\begin{array}{l}40 \\
\quad 67.7 \\
\end{array}$ & & & 59 \\
\hline $\mathrm{Lg}$ & & & & $\begin{array}{l}6 \\
\quad 54.5 \\
\end{array}$ & & $\begin{array}{ll} & \\
& \\
& 45.5 \\
\end{array}$ & & & 11 \\
\hline Gn & & & & $\begin{array}{r}61 \\
\quad 23.3 \\
\end{array}$ & & $\begin{array}{l}200 \\
\quad 76.6 \\
\end{array}$ & & & 261 \\
\hline & 16 & 65 & 28 & 2.623 & 5 & 256 & 19 & 15 & 3.027 \\
\hline
\end{tabular}

Tabla I.-Número de parejas nidificantes (vértice superior) y porcentajes de éstas (vértice inferior) de cada una de las siguientes especies: Fulica atra $(\mathrm{Fa})$, Gallinula chloropus (Gc), Anas platyrhynchos (Ap), Phoenicopterus ruber ( $\mathrm{Pr})$, Charadrius alexandrinus $(\mathrm{Ca})$, Himantopus himantopus $(\mathrm{Hh})$, Recurvirostra avosetta $(\mathrm{Ra})$, Tringa totanus (Tt), Larus ridibundus $(\mathrm{Lr})$, Larus genei $(\mathrm{Lg})$ y Gelochelidon nilotica $(\mathrm{Gn})$. 


\section{III.1. Ocupación. Diversidad y dominancia}

Como consecuencia de la convergencia intraespecífica en las diferentes áreas, se produjo una mayor utilización de unas que de otras. Esta circunstancia se puede observar en el caso concreto del espigón central (área 4). En ella, Phoenicopterus ruber constituyó casi la totalidad de aves reproductoras (Tabla I), lo que provocó que aún presentando la mayor riqueza específica (siete especies) se diese la menor diversidad (Tabla II), con una equitabilidad igualmente baja y con falta de especie subdominante, siendo la especie dominante el Flamenco común.

A continuación de ésta, el área que presentó mayor riqueza específica fue la desembocadura del Arroyo los Arenales (área 7). Por lo tanto se trataba del único punto de agua no salobre (Sánchez, 1984), que perdura en el entorno. Esta superficie fue la que mayor diversidad mostró, careciendo de especie dominante y siendo Recurvirostra avosetta e Himantopus himantopus las especies subdominantes.

\section{TABLA II}

\begin{tabular}{llllc}
\hline & \multicolumn{1}{c}{$\mathbf{H}^{\prime}$} & \multicolumn{1}{c}{ H } & D & SD \\
\cline { 2 - 5 } ÁREA 1 & 0.0 & 0.0 & AP & - \\
ÁREA 2 & 0.857 & 0.62 & CA & - \\
ÁREA 3 & 1.139 & 0.82 & CA & RA \\
ÁREA 4 & 0.246 & 0.13 & PR & - \\
ÁREA 5 & 0.670 & 0.97 & - & CA \\
ÁREA 6 & 0.710 & 0.51 & GN & - \\
ÁREA 7 & 1.660 & 0.92 & - & RA,HH \\
ÁREA 8 & 1.480 & 0.91 & - & FA,HH
\end{tabular}

Tabla II.-Diversidad (H'), Equitabilidad (H), especie dominante (D) y subdominante (SD) de cada área descrita.

Por su parte, los espigones del NE (área 6) fueron los que presentaron el segundo acúmulo de parejas reproductoras (Tabla I), debido a que allí se asentaron las colonias de Láridos, de los que Gelochelidon nilotica supuso el mayor contingente $(76.6 \%$ ), tratándose además de la especie dominante.

$\mathrm{El}$ resto de las áreas presentaron valores cualitativos y cuantitativos inferiores, siendo de destacar que en la orla de la laguna (área 2) Charadrius alexandrinus fue la especie dominante, al igual que lo fue en los espigones del NO. El área 1 fue ocupada solamente por Anas platyrhynchos.

\section{III.2. Segregación interespecífica y amplitud de hábitat}

Observando la figura 2, dendrograma correspondiente al solapamiento específico, se detectan dos grandes grupos; por un lado Láridos, Flamenco y Avo- 


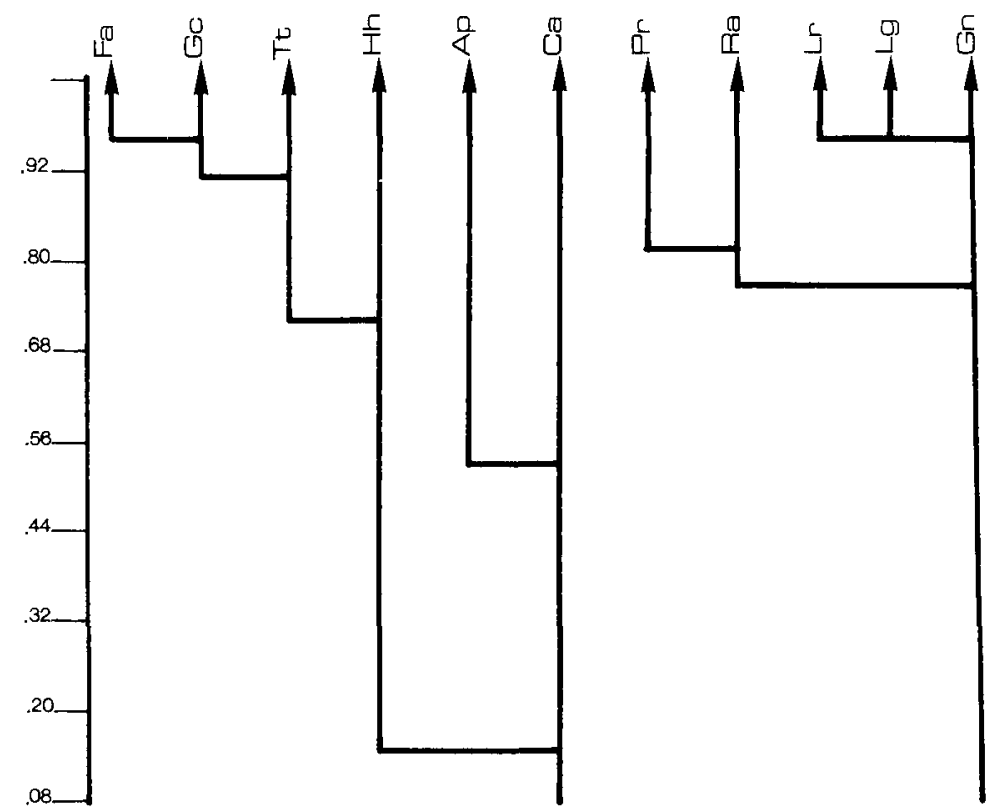

Figura 2.-Dendrograma correspondiente al solapamiento obtenido por el índice de Cody (1974), para las áreas de nidificación de las diferentes especies.

ceta. Por otro el resto de las especies. En el primero, aún formando un grupo compacto, se diferencian dos subgrupos; el formado por Phoenicopterus ruber, especie que muestra una amplitud de hábitat $(\mathrm{AH})$ muy restrictiva (Tabla III) y Recurvirostra avosetta, la especie que presenta mayor amplitud de hábitat. Los láridos, a pesar de poseer amplitudes de hábitat muy pequeños, presentan solapamientos superiores al $95 \%$ y supone el otro elemento de este grupo, a pesar de poseer amplitudes muy pequeñas.

El segundo de los grupos es más heterogéneo tanto por las especies que lo componen (Rálidos, Limícolos y Anátidas) como por el solapamiento que

\section{TABLA III}

\begin{tabular}{llll}
\hline Especies & AH & Especies & AH \\
\hline Fulica atra $\ldots \ldots \ldots \ldots \ldots \ldots \ldots \ldots$ & 1.00 & Recurvirostra avosetta $\ldots \ldots \ldots$. & 4.14 \\
Gallinula chloropus $\ldots \ldots \ldots \ldots \ldots$ & 1.75 & Tringa totanus $\ldots \ldots \ldots \ldots \ldots \ldots \ldots$ & 1.96 \\
Anas platyrhynchos $\ldots \ldots \ldots \ldots \ldots$ & 3.92 & Larus ridibundus $\ldots \ldots \ldots \ldots \ldots$. & 2.16 \\
Phoenicopterus ruber $\ldots \ldots \ldots \ldots$ & 1.00 & Larus genei $\ldots \ldots \ldots \ldots \ldots \ldots \ldots \ldots$ & 1.99 \\
Charadrius alexandrinus $\ldots \ldots$ & 2.42 & Gelochelidon nilotica $\ldots \ldots \ldots \ldots$ & 1.72 \\
Himantopus himantopus $\ldots \ldots$ & 3.77 & & \\
\hline
\end{tabular}

Tabla III.-Valor de la amplitud de hábitat (AH) (Isenmann, 1979) para cada especie estudiada. 
muestran entre ellas. En él se detectan dos grupos que presentan un bajo solapamiento (0.17) entre sí. Por una parte, Anas platyrhynchos y Charadrius alexandrinus y por la otra, Himantopus himantopus y los Rálidos. Los dos primeros se reproduce en un elevado número de áreas, mientras los segundos, a excepción de la Cigüeñuela, lo hacen básicamente en las áreas siete y ocho y presentan una baja amplitud de hábitat.

\section{SINCRONISMO REPRODUCTOR INTERESPECÍFICO}

En la tabla IV se pueden observar los diferentes valores alcanzados por las variables utilizadas para la tipificación de la fenología de las especies. Se observa una serie de especies, que aparecen en el entorno nada más que éste empieza a obtener agua, correspondiendo a Rálidos, Anas platyrhynchos y Phoenicopterus ruber. Los Limícolas a excepción de Himantopus himantopus, tienen también importantes contingentes de invernantes, apareciendo en los meses de enero y febrero. El resto aparecieron en las proximidades del período vernal. De la figura 3, dendrograma correspondiente al solapamiento de la fenología reproductora, se desprende la existenca de dos grupos claramente diferenciados; el primero formado por Fulica atra, Phoenicopterus ruber y Láridos, mientras el segundo lo componen el resto de las especies. En general, se detecta que el solapamiento de las diferentes especies es bajo, incluso en aquellas que muestran mayor solapamiento, cual son los binomios Flamenco comúnPagaza piconegra y Ánade real-Chorlitejo patinegro.

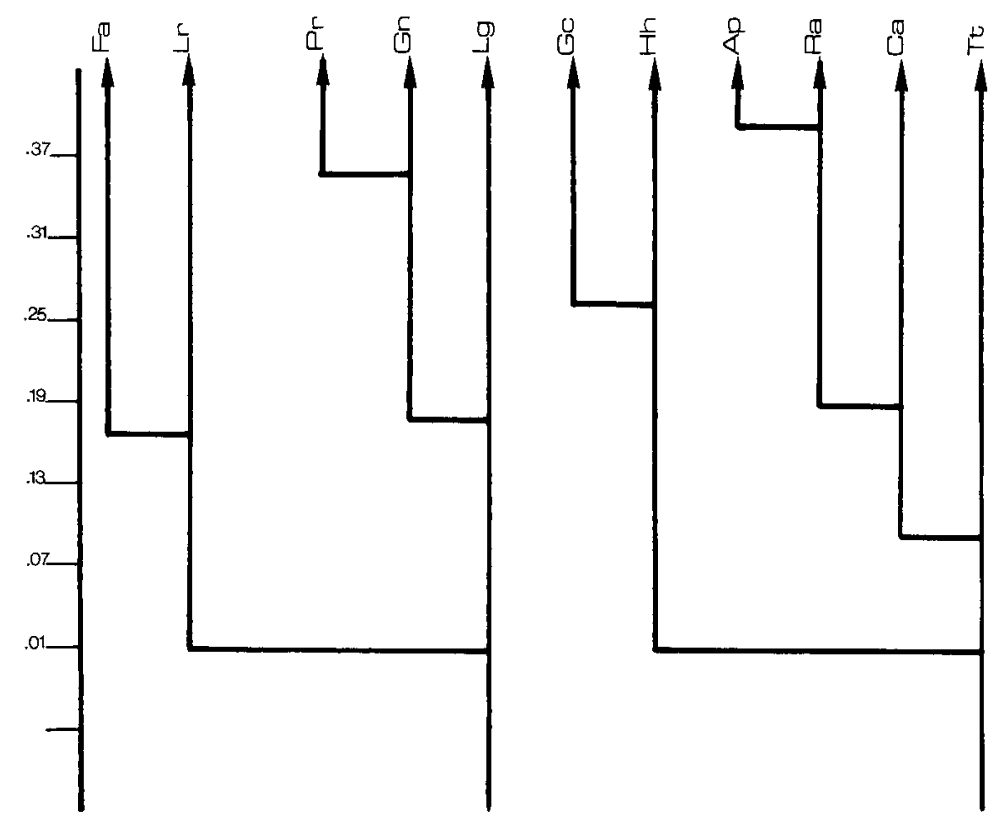

Figura 3.-Dendograma correspondiente al solapamiento fenológico del período reproductor, obtenido por el índice de Pianka (Lawlor, 1980) para las diferentes especies. 


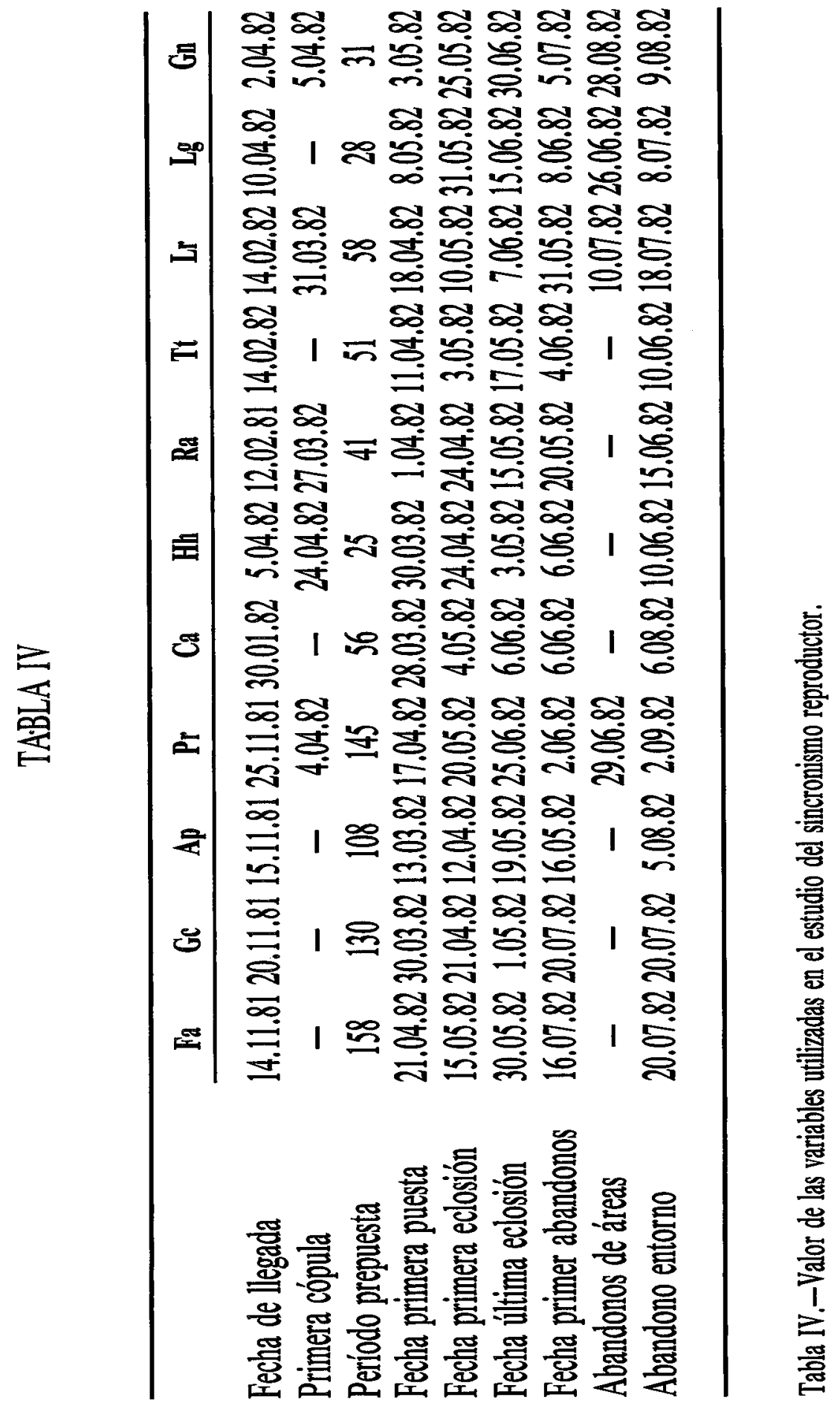




\section{DISCUSIÓN}

Los resultados nos muestran que aquellas especies con mayor sociabilidad (Erwin, 1978), tendieron a provocar importantes aglomeraciones monoespecíficas en aquellas áreas que mayor bondad presentaron. Esta monopolización resultó clara en los casos de Phoenicopterus ruber en el espigón central (área 4) y Gelochelidon nilotica en los espigones del NE (área 6). Esto provocó que sean precisamente esas áreas $(4$ y 6 ) las que mayor densidad de parejas nidificantes presentaron. Además según la bibliografía consultada (Leveque, 1960; Studer-Thiersch, 1968; Ferrer et al, 1976; Antúnez et al, 1977, 1979; Blasco et al, 1979; Vargas et al, 1983; Sánchez, 1984; Sánchez et al, 1985a, b) parece darse una competencia por dichas áreas, en especial la cuatro, ya que los años que no nidifica el Flamenco común en el entorno, dicha área es ocupada por una importante colonia de Pagazas piconegras (Sánchez, 1984) y Carádridos.

La razón de la mayor bondad del espigón central y espigones del NE, para dichas especies, habría que buscarla en su inaccesibilidad, ya que se encuentran en el centro de la masa de agua, permaneciendo rodeadas de ésta hasta avanzado el estío. Por otra parte el componente vegetal es pobre, lo que aumentaba la visibilidad de las parejas respecto a depredadores potenciales (Pedrocchi, 1975), siendo esta vegetación, en especial la de los espigones del NE, lo suficientemente consistente como para dar protección a los pollos de los Láridos, frente a la fuerte insolación que afectaba al entorno en aquellos momentos.

$\mathrm{La}$ asociación o segregación interespecífica (figura 2), muestra la existencia de dos grupos diferenciados. Por una parte, las especies que buscan los islotes y espigones de la masa de agua salobre (Láridos, Flamenco y Avoceta), siendo necesario resaltar la confluencia de algunas asociaciones interespecíficas definidas por diversos autores en otras regiones; así en el Norte de Europa, Jensen (1946), Gloe (1974) y Moller (1982) señalan la coexistencia de Larus ridibundus y Gelochelidon nilotica, mientras en el Oeste las golondrinas de mar cohabitan con Larus genei (Borodulina, 1966; Lehman, 1974; Zubakin-Kostin, 1977). Finalmente, en el Sur de Marruecos y Argelia a esta última asociación se les agregan también los Recurviróstridos (Naurois, 1959; Robin, 1966a, b). Por lo tanto, lo observado en la laguna de Fuente Piedra parece ser una coincidencia de las tendencias mencionadas, coexistiendo las especies que componen las anteriores.

El otro grupo lo componen la única Anátida que se reproduce habitualmente (Vargas et al, 1983; Sánchez, 1984), Limícolas y Rálidos. A excepción de Charadrius alexandrinus, el resto de los componentes son especies que rehuyen generalmente las áreas salobres, siendo de resaltar la segregación que se produce entre los dos Recurviróstidos y que parece extenderse a toda la cuenca endorreica de Fuente Piedra (Studer-Thierrch, 1968; Sánchez, 1982; Vargas et al. 1983). La coexistencia de Anas platyrhynchos y Charadrius alexandrinus con el resto de los miembros de esta asociación, quizás sea más una consecuencia de su amplitud de hábitat que una relación tangible. Dentro de este grupo el conjunto de elementos más compacto es el formado por los Rálidos y Tringa totanus, que aún presentando amplitudes de hábitat pequeñas, presentan solapamientos superiores al $90 \%$. La razón de ello parece hallarse en su clara predilección por las aguas dulces, que es aún mayor que en el resto de los componentes de esta asociación. 
De los resultados obtenidos para el sincronismo reproductor, se observa que éste, en cualquiera de los casos, es pobre, siendo siempre inferior al $40 \%$, detectándose dos grupos que quedan segregados a su vez en dos subgrupos cada uno. El primer grupo, constituido por Láridos, Flamenco y Focha común, viene definido por especies que muestran un retraso en el inicio de su reproducción respecto del resto (segunda quincena de abril y primera de mayo). Se trata de un grupo de especies que muestran como denominador común el reproducirse, ya sean de agua dulce o salobre, sobre las áreas emergidas más inaccesibles del entorno y que permanecen rodeadas de agua hasta avanzado el verano. Probablemente a estas especies ligadas al agua, el avance del estiaje del entorno les sea menos acuciante. Además, la segregación que dentro del grupo se detecta, agrupa por un lado las poblaciones no migradoras (Fulica atra y Larus ridibundus) (Vargas et al. 1983; Sánchez, 1984) y por otro las migradoras, ya que si bien Phoenicopterus ruber es una de las primeras especies en aparecer, es en bajo número y según parece, se trata de formas que se reproducen más al Norte (Camarga, Francia) (Blasco et al., 1979).

La única característica que hemos encontrado como común a los componentes del otro grupo es, que aún siendo especies que nidifiquen en masas de agua salobres o no, lo suelen hacer en las porciones del perímetro de las masas de agua, excepción hecha de Recurvirostra avoseta que más de la mitad lo hacen en los espigones del NE y Central. Pero esta especie es la migradora de aparició más temprana, lo que podría provocar su adelanto en el inicio de su ciclo reproductor y su inclusión en este grupo. 


\section{BIBLIOGRAFÍA}

AMAT, J. A. 1984: Las poblaciones de aves acuáticas en las Lagunas andaluzas: composición y diversidad durante un ciclo anual. Ardeola 31: 61-79.

ANTÚNEZ, A.; VARGAS, J. M. y BLASCO, M. 1977: El comportamiento reproductivo y alimentario de la Pagaza Piconegra (Gelochelidon nilotica. Gm) en la laguna de Fuente Piedra. Ardeola 25: 227-231.

ANTÚNEZ, A.; BLASCO, M. y VARGAS J. M. 1979: Informe sobre Phoenicopterus ruber en la laguna de Fuente Piedra 1978. Mon. Trab. Dpto. Zool. 1: 1-17.

BLASCO, M.; LUCENA, J. y RODRÍGUEZ, J. 1979: Los flamencos de Fuente Piedra. Naturalia Hispánica. 23. ICONA Madrid.

BORODULINA, T. L. 1988: Biology and economic importance de gulls and terns of southern USSR water bodies. Israel Prog. Sicientif. Translations. Jerusalem.

CODY, M. 1974: Competitions and the structures of bird comunities Princeton. Ed. 318 pág.

ERWIN, R. M. 1978: Coloniality in ters: the role of social feeding Condor 80: 211-215 pág.

FERRER, J.; GARCÍA, L. y PURROY, F. J. 1976: Informe sobre el flamenco y su población en 1974. Bol. Central Ecol. 5: 55-72.

GLOE, P. 1974: Die Lachseeschwalbe (Gelochelidon nilotica) in Ditmarschen. Vogelwelt 95: 4751 pág..

ISENMANN, P. V. 1979: Le partage des biotopes de Camargue por les larides nicheurs. L'oiseau et R. F. O. 49: 1972 (2).

JENSEN, P. V. 1946: Nogles iagttagelser over Sandterness (Gelochelidon nilotica. Gm) biologi Dansk. Norn. For. Tiddkr. 40: 80-95 pág.

LAWLOR, L. R. 1980: Overlap, similarity, and competition coefficients. Ecology 6 (2): 245-251.

LEHMAN, H. 1974: Brutkolonien im Hochland zentralantatlies Verein Wuppertal 27: 80-104 pág.

LEVEQUE, R. 1960: Una visita a la laguna de Fuente Piedra. Ardeola 6:344-347 pág.

MOLLER, A. P. 1982: Coloniality and colony structure in Gull-billed tern /Gelochelidon nilotica). J. Ornith. 123: 41-53,

NAUROIS, R. 1959: Premieres recherche sur l'avifaune des iles du Banc d'Argui (Mauritania). Alauda 27:241-308 pág.

PEDROCCHI, C. 1975: Efecto topoclimático en la densidad de nidificación de aves. P. Cent. Pir. Biol. Exp. 7(2): 163-167.

ROBIN, A. P. 1966a: Nidifications sur L'iriki, Daya temporaire de Sud Marocain, en 1965. Alauda, 34: 81-101 pág.

- 1966b: L'avifauna de L'iriki (Sud-Marocain). Alauda 36: 237-253 pág.

SÁNCHEZ, J. M. 1982: La avifauna de la laguna dulce de Campillós (Málaga). Mon. Trab. Zool., 3-4: 84-103 pág.

- 1984: Contribución al conocimiento de la Biología y desarrollo de Gelochelidon nilotica (Gm., 1789). Tesis Doctoral. Universidad de Málaga. 567 pág.

SÁNCHEZ, J. M.; BLASCO, M. y ROSADO, L. M. 1981: Los vertebrados de la laguna dulce de Campillos. Bol. Estación Cen. Ecol. 10: 109-115.

SÁNCHEZ, J. M.; VARGAS, J. M. y BLASCO, M. 1985: Historia y evolución de la colonia de flamenco de la laguna de Fuente Piedra. Bol. Estación Cent. Ecol. 28: 9-18 pág.

SÁNCHEZ, J. M.; BLASCO, M. y DA SILVA, E. 1985a: Evolución y situación actual de los Láridos nidificantes en la laguna de Fuente Piedra (Málaga). VIII. J. O. E. 19 pág.

SÁNCHEZ, J. M. y DE LA CRUZ, C. 1985b: Sobre la reproducción del Flamenco común en la laguna de Fuente Piedra en 1984 y algunas consideraciones sobre dicha población VIII. J. O. E. 15 pág.

SHANNON, C. E. 1948: A mathematical theory of comunication. Boll. System. Fech. J., 27:, 379-423 pág.

STUDER-THIERSCH, A. 1968: Sobre aves acuáticas en una laguna del interior de Andalucía. Ardeola 14: 166-174 pág.

THEVENOT, M. 1982: Contribution a l'etude ecologique des Passereux forestiers du Plateu Central et de la corniche du Moyen Atlas (Maroc). L'oiseaux et R. F. O. 1982 (2): 97-152.

VALVERDE, J. A. 1964: La reproducción de flamencos en Andalucía en el año 1963. Ardeola 9: $55-65$.

VARGAS, J. M.; BLASCO, M. y ANTÚNEZ, A. 1983: Los Vertebrados de la laguna de Fuente Piedra (Málaga). Monografía (28): I.CO.NA Madrid. 228 pág.

ZUBAKIN, V. A. y KOSTIN, Y. V. 1977: Breeding birds of the Changarskije Is. Ornithologija 13: 49-55 pág. 